\title{
Comment on "EM induction in elongated conductors normal to a coastline with application to geomagnetic measurements in Nigeria" by J. Chen, H. W. Dosso and S. Kang
}

\author{
P. F. Chen \\ Department of Physics, University of Hong Kong, Hong Kong, China
}

(Received August 24, 1998; Revised March 2, 1999; Accepted June 22, 1999)

\section{On the Frequency Response of Induction Ar- rows}

The induction frequency response of some analogue models shown in Chen, Dosso and Kang (1997; hereafter referred to as CDK), seems to be somewhat strange. The frequency behaviour of the in-phase and quadrature Parkinson arrows, shown in figures 2 and 4 of CDK, indicates that: 1) the magnitude of the in-phase arrow decreases rapidly with increasing period, and becomes negligible when $T=30 \mathrm{~min}$; 2) the quadrature arrow does not change its sign in the studied inducing period from $1 \mathrm{~min}$ to $90 \mathrm{~min}$. In order to examine the correctness of the experimental results for their models, I have carried out some 3-D numerical modelling calculations using the finite difference method. I shall devide this comment into two parts. The first part gives the numerical results of an example, a model suggested by Wannamaker et al. (1984) for demonstrating the applicability of the finite difference method. The second part shows my numerical results for the elongated parallel conductors model mentioned in CDK, indicating that their analogue result is suspect.

\section{The 3-D Finite Difference Method}

The outline of the 3-D finite difference method and the detailed algorithm can be found in Chen (1985) and Chen and Fung (1988). The accuracy obtained by our computer program has been tested by making calculations for Weidelt's model (Weidelt, 1975) and Ting and Hohmann's model (Ting and Hohmann, 1981). The comparisons of our numerical results and their results are shown in the Appendix of Chen and Fung (1988). Another comparison for Hohmann's model (Hohmann, 1983) has also been carried out (Chen and Fung, 1989), and in that model the conductivity contrast is 200. All the results obtained by different methods agree well for the most part of the response curves.

Here I show another comparison with the third model constructed by Wannamaker et al. (1984). In the stated model the conductive body is a plate-like feature $1 \mathrm{~km}$ thick at a depth of $750 \mathrm{~m}$, with the lower layer situated below $2250 \mathrm{~m}$. Its width is $5 \mathrm{~km}$ and its strike length is $60 \mathrm{~km}$ (see Fig. 1). The mesh used in this model consists of $85,995(91 \times 35 \times 27)$

Copy right $(\mathrm{C}$ The Society of Geomagnetism and Earth, Planetary and Space Sciences (SGEPSS); The Seismological Society of Japan; The Volcanological Society of Japan; The Geodetic Society of Japan; The Japanese Society for Planetary Sciences. grid points; the grid spacings in the central part of the model are $0.25 \mathrm{~km}$ in the $y$-direction, $3.0 \mathrm{~km}$ in the $x$-direction, and the grid spacings in the upper part of the $z$-direction are 0.25 $\mathrm{km}$. My numerical results are shown in Fig. 2. Following Wannamaker et al.'s notation $H_{z s}^{o}$ is the scattered part of $H_{z}$ at the ground surface; $H_{y i}$ is the incident source field. The inducing frequency is $1.0 \mathrm{~Hz}$. The solid lines in Fig. 2 are the 2-D traverse results. The 3-D numerical results of $H_{z s}^{o} /\left|H_{y i}^{o}\right|$ along the central traverse are almost the same as the 2-D results for the in-phase part, but the 3-D and 2-D results do not agree well for the quadrature part (see Fig. 2a). Accordingly, the 3-D and 2-D results of the quadrature induction arrow do not agree well either (see Fig. 2b). Nevertheless, it is worth noting that, if we reverse the signs both for obtaining the in-phase and quadrature arrows, the quadrature Parkinson arrows in this example $(T=1 \mathrm{sec})$ point away from the conductor. As a matter of fact, when $T \geq 7 \mathrm{sec}$, the quadrature Parkinson arrows will change their directions; in that case, they will point towards the conductor.

\section{The Elongated Parallel Conductors Model with- out the Ocean}

Figure 1 of CDK shows the structure of the model and figure 2 of CDK shows the response of $V_{y}$ (the response of the elongated conductors without the ocean). There is a pair of surface elongated conductors $(3.6 \mathrm{~S} / \mathrm{m})$ : both conductors' lengths and thicknesses are $500 \mathrm{~km}$ and $5 \mathrm{~km}$ respectively, the width is $20 \mathrm{~km}$ for conductor $\mathrm{b}$ and $5 \mathrm{~km}$ for conductor a. The conductor separation distance $(S)$ is $50 \mathrm{~km}$. The conductivity of the first layer is $6.25 \times 10^{-4} \mathrm{~S} / \mathrm{m}$, and a highly conductive layer $(3.6 \mathrm{~S} / \mathrm{m})$ lies below $200 \mathrm{~km}$. The conductivity contrast in this model is very high. This high contrast condition leads to the convergence of the iteration process in FDM becoming very slow. However, this is not a serious difficulty. I have examined the calculations by using different grid spacings in the $x-, y$ - and $z$-directions and have proved that the iteration is convergent and the results are stable. In this model calculations (note that the ocean is absent here), the magnetic field components are calculated in the $X$-polarization case for traverses in the $y$-direction. It implies that the induction arrows are calculated simply as $H_{z} / H_{y}$. This treatment is acceptable when $H_{x}$ is much smaller than $H_{y}$. As my understanding, CDK used the same 
PLAN VIEW

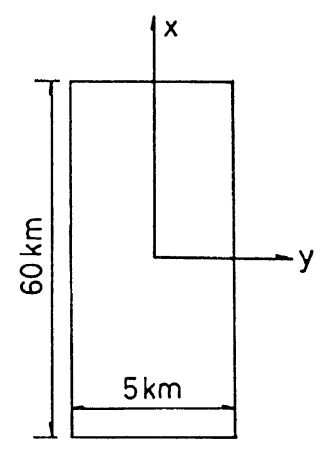

SECTION VIEW

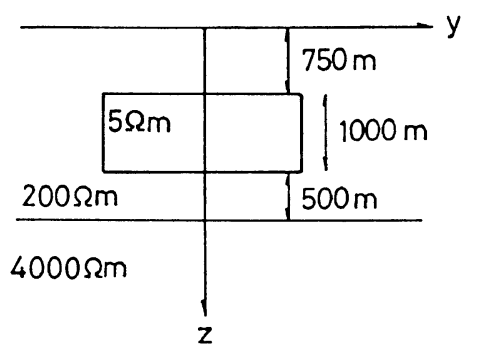

Fig. 1. The model constructed by Wannamaker et al. (1984).
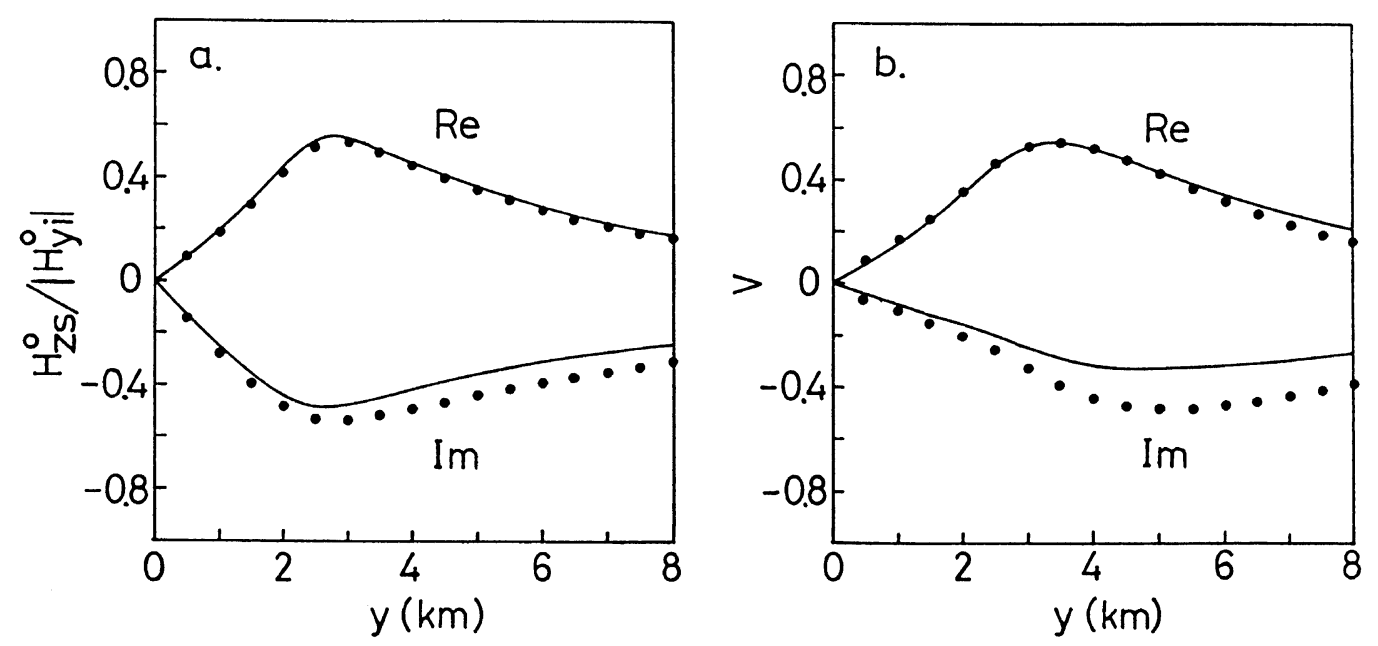

Fig. 2. The numerical results obtained by the 3-D finite difference method for the model described in Fig. 1 . a) The vertical magnetic components $H_{z s}^{o} /\left|H_{y i}^{o}\right|$ along the central traverse; b) The induction arrows $\mathrm{V}$ along the central traverse (here both the real and imaginary arrows are not reversed in sign). The solid lines are the 2-D results for comparison. The filled dots are the 3-D numerical results obtained by FDM.

definition as stated above (see the last three lines in page 1402 of CDK's paper).

Figure 3 shows the frequency response of the in-phase and quadrature Parkinson arrows along traverse T2 $(30 \mathrm{~km}$ from the ends of the conductors). The difference of the in-phase arrows between the numerical and analogue results at longer periods is very clear. The numerical results indicate that a significant in-phase induction arrow can be found even at the longer period of $T=60 \mathrm{~min}$ (see the solid lines in Fig. 3). The dotted lines are the analogue results obtained by CDK (reproduced from figure 2 of CDK's paper).

The second point is that a characteristic period, about 5 min for conductor $b$, exists in the numerical results; outside the conductor b (say $15 \mathrm{~km}$ outside the left interface of conductor $b$ ) the quadrature induction arrow changes its sign when the period passes the characteristic period. Similar behaviour can also be found for conductor a (outside the right interface of conductor a). This behaviour is similar to that in the 2-D case. In accordance with CDK's definition, both the real and imaginary arrows are reversed in sign for the time varying field of the form $\exp (i \omega t)$. Therefore, at an observation point beyond the interface (but not very near the interface) of the conductor, the quadrature arrow points towards the conductor at longer periods and points away from the conductor at shorter periods. However, the sign change in quadrature induction arrows is absent in CDK's analogue results.

Furthermore, let us see another comparison in order to demonstrate clearly the discrepancy between the analogue and numerical results. Figure 4 shows the period response curves for $S=50 \mathrm{~km}$ at selected sites $(-Y)$ between the pair of parallel conductors for the model shown in Fig. 3. The solid lines in Fig. 4 are the numerical results and the dashed lines are the analogue results (reproduced from figure 4 of $\mathrm{CDK})$. At sites $(Y=-45$ and $-40 \mathrm{~km})$ near conductor $\mathrm{b}$, the quadrature responses are positive at the very short periods (1 and $2 \mathrm{~min}$ ) (see the solid lines) and are negative at longer periods. However, the quadrature responses obtained 

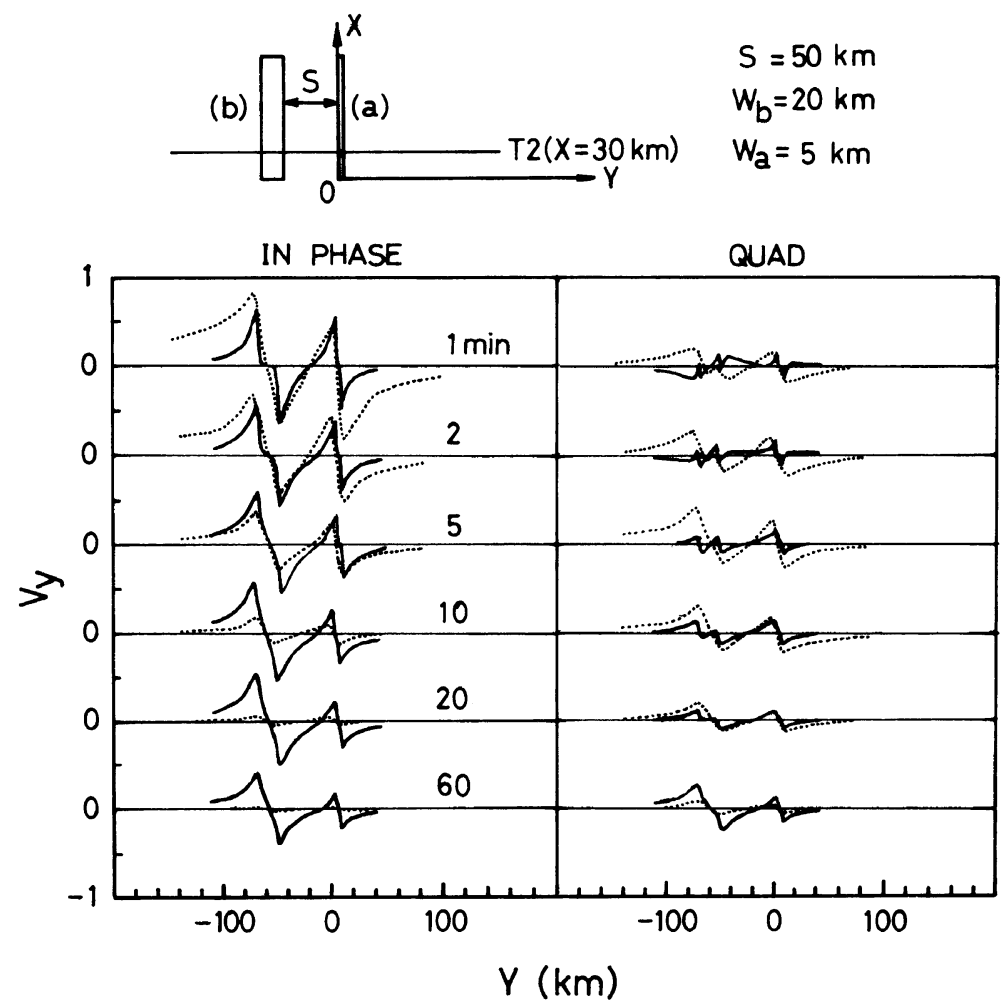

Fig. 3. The in-phase and quadrature induction arrow $V_{y}$ for $\mathrm{T} 2$ over the model of a pair of elongated conductors (without the ocean) for $W_{\mathrm{a}}=5 \mathrm{~km}$, $W_{\mathrm{b}}=20 \mathrm{~km}$ and $S=50 \mathrm{~km}$. The solid lines are the numerical results. The dotted lines are the analogue results (reproduced from figure 2 of CDK's paper).

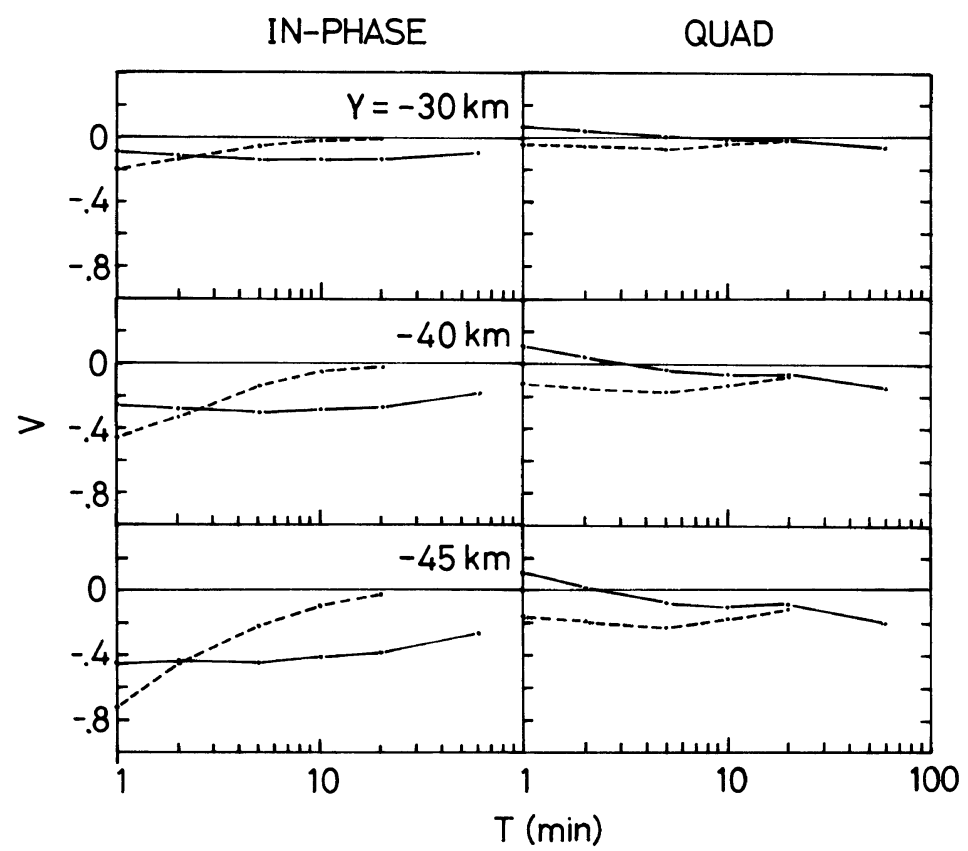

Fig. 4. The comparison of the frequency responses of the in-phase and quadrature arrows $V$ at selected sites $(-Y)$ between the elongated parallel conductors while the conductor separation distance is $50 \mathrm{~km}$. The solid lines are the numerical results. The dashed lines are the analogue results (reproduced from figure 4 of CDK's paper). 
by $\mathrm{CDK}$ are negative at all periods at the stated sites (see the dashed lines). Besides, the rapid decrease of the in-phase arrows with increasing period does not appear in the numerical results. On the contrary, a maximum magnitude of in-phase arrows can be found when $T$ is near the characteristic period. This feature is also similar to that in the 2-D case.

Based on the results above, I consider that something must be wrong with the analogue measurements carried out by CDK. The rapid decrease of the in-phase Parkinson arrow with increasing period, shown in CDK, is suspect. The behaviour of the quadrature induction arrow, i.e. no sign change can be found in CDK's results, is also suspect.

In order to justify the FDM results shown here, I hope that numerical modelling using other methods, such as the finite element method, the integral method or the hybrid method, of the model mentioned above can be carried out by other workers in the near future.

Acknowledgments. The writer would like to thank two referees, Prof. P. Weidelt and Dr. J. D. McKnight, for their valuable comments and helpful suggestions.

\section{References}

Chen, J., H. W. Dosso, and S. Kang, EM induction in elongated conductors normal to a coastline with application to geomagnetic measurements in NIGERIA, J. Geomag. Geoelectr., 49, 1401-1414, 1997.

Chen, P. F., On the finite difference method of three-dimensional electromagnetic problem, Acta Geophysica Sinica, 28, 268-281, 1985 (in Chinese).

Chen, P. F. and P. C. W. Fung, Frequency response of the transfer functions of the current channelling between two oceans, J. Geomag. Geoelectr., 40, 335-355, 1988

Chen, P. F. and P. C. W. Fung, A mesh convergence test for the finite difference method in three-dimensional electromagnetic modelling, Chinese J. Geophys., 32(4), 569-573, 1989.

Hohmann, G. W., Three-dimensional EM modeling, Geophys. Surv., 6, 2753, 1983.

Ting, S. C. and G. W. Hohmann, Integral equation modelling of threedimensional magnetotelluric response, Geophysics, 46, 182-197, 1981.

Wannamaker, P. E., G. W. Hohmann, and W. A. San Filipo, Electromagnetic modeling of three-dimensional bodies in layered earths using integral equations, Geophysics, 49, 60-74, 1984.

Weidelt, P., Electromagnetic induction in three-dimensional structures, $J$. Geophys., 41, 85-109, 1975.

P. F. Chen (e-mail: hrspcpf@hkucc.hku.hk) 